\title{
High-fidelity conversion of photonic quantum information to telecommunication wavelength with superconducting single-photon detectors
}

\author{
Rikizo Ikuta, ${ }^{1}$ Hiroshi Kato, ${ }^{1}$ Yoshiaki Kusaka, ${ }^{1}$ Shigehito Miki, ${ }^{2}$ Taro Yamashita, ${ }^{2}$ Hirotaka Terai, ${ }^{2}$ \\ Mikio Fujiwara, ${ }^{3}$ Takashi Yamamoto, ${ }^{1}$ Masato Koashi, ${ }^{4}$ Masahide Sasaki, ${ }^{3}$ Zhen Wang, ${ }^{2}$ and Nobuyuki Imoto ${ }^{1}$ \\ ${ }^{1}$ Graduate School of Engineering Science, Osaka University, Toyonaka, Osaka 560-8531, Japan \\ ${ }^{2}$ Advanced ICT Research Institute, National Institute of Information and \\ Communications Technology (NICT), 588-2 Iwaoka, Kobe 651-2492, Japan \\ ${ }^{3}$ Advanced ICT Research Institute, National Institute of Information and Communications Technology (NICT), \\ 4-2-1 Nukuikawa, Koganei, Tokyo 184-8795, Japan \\ ${ }^{4}$ Photon Science Center, The University of Tokyo, Bunkyo-ku, 113-8656, Japan
}

\begin{abstract}
We experimentally demonstrate a high-fidelity visible-to-telecommunication wavelength conversion of a photon by using a solid-state-based difference frequency generation. In the experiment, one half of a pico-second visible entangled photon pair at $780 \mathrm{~nm}$ is converted to a $1522-\mathrm{nm}$ photon, resulting in the entangled photon pair between $780 \mathrm{~nm}$ and $1522 \mathrm{~nm}$. Using superconducting single-photon detectors with low dark count rates and small timing jitters, we selectively observed well-defined temporal modes containing the two photons. We achieved a fidelity of $0.93 \pm 0.04$ after the wavelength conversion, indicating that our solid-state-based scheme can be used for faithful frequency down-conversion of visible photons emitted from quantum memories composed of various media.
\end{abstract}

Wavelength conversion of photons in a quantum regime [1] has been actively studied [2 [10] as a quantum interface for application of quantum information processing and communications. Especially, such a conversion aiming at near-infrared photons in telecommunication bands are essential for transmitting quantum information over long-distance optical fiber networks with quantum repeaters [11 13]. In the quantum repeaters, the photon sent to a relay point through an optical fiber needs to be entangled with a quantum memory. At present, many of quantum memories and processors based on alkaline atoms, trapped ions and solid states have successfully created entanglement with photons at around visible wavelengths 14 20]. Thus, a quantum interface for the wavelength conversion from visible to telecommunication bands with a high fidelity has attracted much interest for its applications. So far such a quantum interface has been demonstrated by using four wave mixing with a cold atomic cloud [6] or difference frequency generation (DFG) from a nonlinear optical crystal [7]. Among them, nonlinear optical crystals with waveguide structure have practically desirable features. They can operate near room temperature and do not require laser cooling configuration, enabling a compact setup and integration into a photonic quantum circuit on a chip using waveguide structures [21]. In addition, they have a wider bandwidth, compatible with wide-band quantum memories 22, 23], resulting in high-clock-rate quantum information processing. Such kind of solid-state-based optical quantum interface lead to development of a mature quantum information technology. However, in several demonstrations of the solid-state-based wavelength conversion [4, 7, 24 26], they suffered from degradation of an observed fidelity of a reconstructed quantum state after the wavelength conversion due to background noises caused by Raman scattering of a strong cw pump light and relatively high dark count rate of an InGaAs/InP avalanche photodiode (APD) for photon detection at the telecommunication band. Therefore the observed fidelity of the state after the wavelength conversion is degraded.

In this Letter, we demonstrate almost noiseless wavelength conversion by suppressing the effects of both the optical noise from the Raman scattering and the dark count via newly developed superconducting singlephoton detectors (SSPDs) for visible and telecommunication wavelengths of the photons 27, 28]. The SSPDs have lower dark count rates and smaller timing jitters than those of typical APDs. Especially, the latter property enables us to selectively observe well-defined temporal modes containing the two photons. Because duration of signal photons in our experiment is of pico-second order whereas the optical noise through the wavelength conversion are continuously generated, the use of the SSPDs will lead to reduction of irrelevant photon detections. The observed fidelity of the two-photon state after the wavelength conversion to a maximally entangled state is $0.93 \pm 0.04$, which is very close to the initial fidelity of $0.97 \pm 0.01$. We also clearly observe the violation of the Clauser-Horne-Shimony-Holt-type Bell's inequality with $S=2.62 \pm 0.09$.

Theoretical treatment of wavelength conversion of a single mode of a pulsed light is as follows [1, 7]. When a pump light at angular frequency $\omega_{\mathrm{p}}$ is sufficiently strong, the Hamiltonian of the wavelength conversion using a second-order nonlinear optical interaction is described by $\hat{H}=i \hbar \sqrt{\eta P}\left(e^{-i \varphi} \hat{a}_{\mathrm{c}}^{\dagger} \hat{a}_{\mathrm{s}}-e^{i \varphi} \hat{a}_{\mathrm{s}}^{\dagger} \hat{a}_{\mathrm{c}}\right)$. Here $\hat{a}_{\mathrm{s}}$ and $\hat{a}_{\mathrm{c}}$ are annihilation operators of a signal mode at angular frequency $\omega_{\mathrm{s}}$ and a converted mode at angular frequency $\omega_{\mathrm{c}}=\omega_{\mathrm{s}}-\omega_{\mathrm{p}}$, respectively. $P$ and $\varphi$ are a power and a phase of the classical pump light, respectively. $\eta$ is a con- 


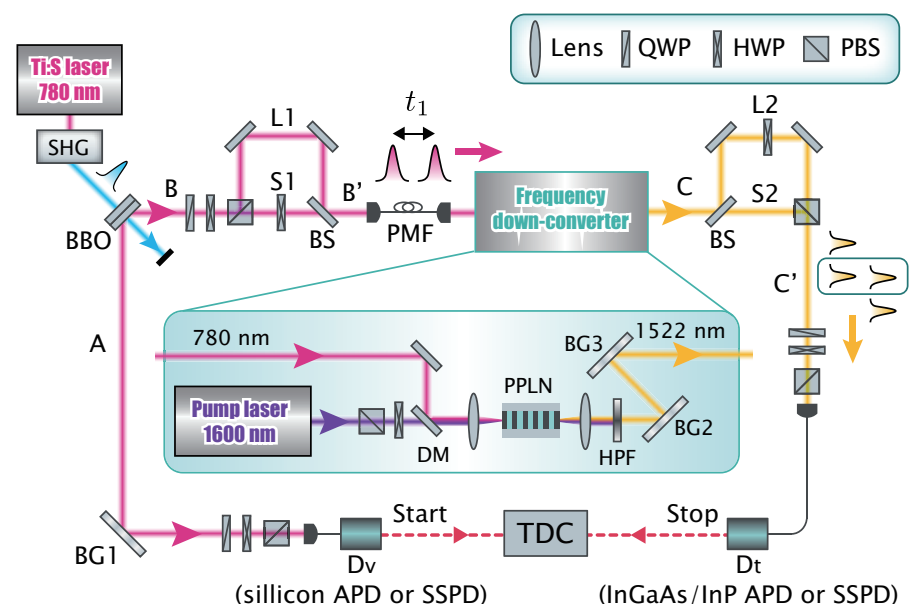

FIG. 1: The experimental setup for frequency downconversion of one halves of the visible entangled photon pairs.

stant and $\sqrt{\eta P} e^{i \varphi}$ represents an effective coupling constant. Using this Hamiltonian, an annihilation operator $\hat{a}_{\mathrm{c}, \mathrm{out}}$ of the converted mode coming from the nonlinear optical crystal is described by using the time evolution of $\hat{a}_{\mathrm{s}}$ in the Heisenberg picture as

$$
\hat{a}_{\mathrm{c}, \text { out }}=\hat{a}_{\mathrm{c}}(\tau)=e^{-i \varphi} \sin (\sqrt{\eta P} \tau) \hat{a}_{\mathrm{s}}+\cos (\sqrt{\eta P} \tau) \hat{a}_{\mathrm{c}},(1)
$$

where $\tau$ is the traveling time of the pulses through the crystal.

The experimental setup for the DFG-based frequency down-conversion of one half of a polarization-entangled photon pair at $780 \mathrm{~nm}$ to the wavelength of $1522 \mathrm{~nm}$ is shown in Fig. 1. We use a mode-locked Ti:sapphire laser (wavelength: $780 \mathrm{~nm}$; pulse width: $1.2 \mathrm{ps;} \mathrm{repeti-}$ tion rate: $82 \mathrm{MHz}$ ) as a light source. It is frequency doubled by a second harmonic generator (SHG), and then the UV pulse with a power of $250 \mathrm{~mW}$ pumps a pair of typeI phase-matched 1.5 -mm-thick $\beta$-barium borate (BBO) crystals to prepare the polarization-entangled photon pair $\mathrm{A}$ and $\mathrm{B}$ which is described as $\left|\phi^{+}\right\rangle_{\mathrm{AB}} \equiv\left(|\mathrm{HH}\rangle_{\mathrm{AB}}+\right.$ $\left.|\mathrm{VV}\rangle_{\mathrm{AB}}\right) / \sqrt{2}$ through spontaneous parametric downconversion [29]. Here $|\mathrm{H}\rangle$ and $|\mathrm{V}\rangle$ represent horizontal $(\mathrm{H})$ and vertical $(\mathrm{V})$ polarization states of a photon. The spectrum of photon A is narrowed by a Bragg grating (BG1) with a bandwidth of $0.2 \mathrm{~nm}$, and then the photon is detected by detector $\mathrm{D}_{\mathrm{v}}$ connected to a singlemode fiber. We switch the silicon APD and the SSPD for $\mathrm{D}_{\mathrm{v}}$. Photon B is split into a short path (S1) and a long path (L1) according to a polarization of the photon. A half-wave plate (HWP) in S1 flips the polarization from $\mathrm{H}$ to $\mathrm{V}$. As a result, a polarization qubit $\{|\mathrm{H}\rangle,|\mathrm{V}\rangle\}$ in mode $\mathrm{B}$ is transformed to a time-bin qubit $\{|\mathrm{S} 1\rangle,|\mathrm{L} 1\rangle\}$ in mode $\mathrm{B}^{\prime}$, leading to a two-photon state in modes $\mathrm{A}$ and $\mathrm{B}^{\prime}$ of $|\psi\rangle_{\mathrm{AB}^{\prime}} \equiv\left(|\mathrm{H}\rangle_{\mathrm{A}}|\mathrm{S} 1\rangle_{\mathrm{B}^{\prime}}+|\mathrm{V}\rangle_{\mathrm{A}}|\mathrm{L} 1\rangle_{\mathrm{B}^{\prime}}\right) / \sqrt{2}$. Here $|\mathrm{S} 1\rangle$ and $|\mathrm{L} 1\rangle$ represent states of $\mathrm{V}$-polarized pho- tons passing through $\mathrm{S} 1$ and L1, respectively. We set the time difference between S1 and L1 to about 700 ps. The photon in mode $\mathrm{B}^{\prime}$ goes through a polarizationmaintaining fiber (PMF) and then enters the frequency down-converter whose details are shown in the inset of Fig. [1]

For the DFG of the signal photon at $780 \mathrm{~nm}$, a cw pump laser at $1600 \mathrm{~nm}$ is used. The linewidth of the pump light is $150 \mathrm{kHz}$, and its coherence time is much longer than the time difference of the photons passing through S1 and L1. The pump light is combined with the signal photon at a dichroic mirror (DM) after its polarization is set to $\mathrm{V}$ by a polarization beamsplitter (PBS) and a HWP. Then they are focused on the type- 0 quasiphase matched $(\mathrm{V} \rightarrow \mathrm{V}+\mathrm{V})$ PPLN waveguide [30] whose temperature is controlled to be about $50^{\circ} \mathrm{C}$. The length of the PPLN crystal is $20 \mathrm{~mm}$ and the acceptable bandwidth is calculated to be about $0.3 \mathrm{~nm}$. After passing through the PPLN waveguide, the strong pump light is diminished by a high-pass filter (HPF), and the light converted to the wavelength of $1522 \mathrm{~nm}$ is extracted by BG2 and BG3 whose bandwidths are $1 \mathrm{~nm}$.

The photon in mode $\mathrm{C}$ from the frequency downconverter is split into a short path (S2) and a long path (L2) by a BS. The polarization of the photon passing through L2 is flipped from V to H by a HWP. Time difference between S2 and L2 is adjusted to be the same as that between S1 and L1. The components of the photon from S2 and L2 are recombined by a PBS, and the photon in mode $\mathrm{C}^{\prime}$ is detected by $\mathrm{D}_{\mathrm{t}}$ after passing through a single-mode fiber. We switch the InGaAs/InP APD and the SSPD for $D_{t}$.

Each SSPD consists of an 100-nm-thick Ag mirror, a $\lambda / 4 \mathrm{SiO}$ cavity and 4-mm-thick niobium nitride meander nanowire on a 0.4 -mm-thick $\mathrm{MgO}$ substrate from the top. The nanowire is 80 -nm-wide, and it covers an area of $15 \mu \mathrm{m} \times 15 \mu \mathrm{m}$. The respective optical cavity structures of the SSPDs for $\mathrm{D}_{\mathrm{v}}$ and $\mathrm{D}_{\mathrm{t}}$ are designed for visible and the telecommunication wavelengths to achieve higher detection efficiencies. The detection efficiencies are $32 \%$ and $12.5 \%$ for $780 \mathrm{~nm}$ and $1522 \mathrm{~nm}$ wavelengths, respectively. Each of the SSPD chip is shielded by a copper block which has a holder of a single-mode optical fiber followed by a small-gradient index lense for efficient coupling. The blocks are installed in the GiffordMcMahon cryocooler system whose cooling temperature is $2.28 \pm 0.02 \mathrm{~K}$.

To measure the coincidence events of the detections at $D_{v}$ and $D_{t}$, signals from $D_{v}$ and $D_{t}$ are input to a timeto-digital converter (TDC) as a start and stop signals of a clock, respectively. By post-selecting the events where the photon in mode $\mathrm{C}^{\prime}$ has passed through S1-L2 or L1S2, we obtain the polarization-entangled state $\left|\phi^{+}\right\rangle_{\mathrm{AC}^{\prime}}$ in modes $\mathrm{A}$ and $\mathrm{C}^{\prime}$. In our experiment, we accept such events in 200-ps time window.

For a faithful wavelength conversion, the rate of back- 


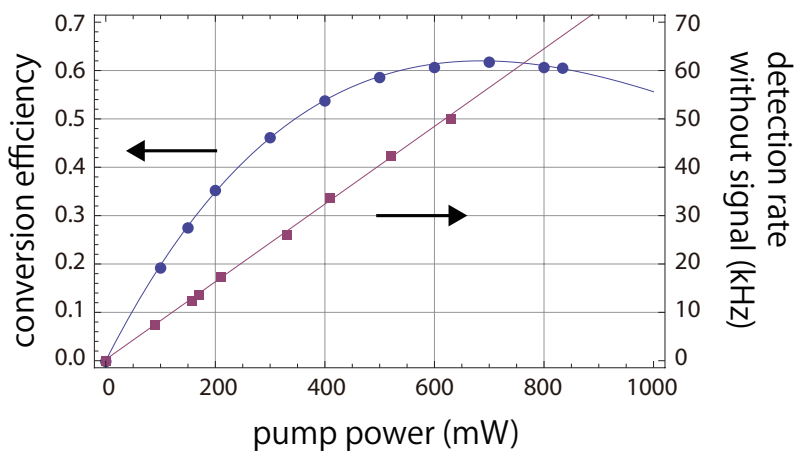

FIG. 2: The dependencies of the conversion efficiency and the rate of the background noises on the pump power. The former has been measured by using a pico-second coherent light in Ref. [7]. The latter was measured by using the SSPD for $\mathrm{D}_{\mathrm{t}}$ without the UV pulse. The first vertical axis is the conversion efficiency. A maximum conversion efficiency is achieved at a pump power of $700 \mathrm{~mW}$. The second vertical axis is detection rate of the background noises. We fitted the experimental data by a function of $b P+d . \quad b$ and $d$ are estimated as 80 $\mathrm{Hz} / \mathrm{mW}$ and $266 \mathrm{~Hz}$, respectively.

ground noises must be sufficiently small compared to that of the converted photons. The background noises are mainly caused by the Raman scattering of the pump light and dark countings of a photon detector. The former is proportional to the pump power and is written by $b P$ with a constant $b$. The latter is described by a constant $d$. Taking into account these noise effects and using Eq. (11), the signal-to-noise ratio for the observed converted photons is represented by

$$
f_{\mathrm{SNR}}(P) \equiv \frac{a \sin ^{2}(\sqrt{\eta P} \tau)}{b P+d}
$$

where $a$ is a constant. When $d$ is comparable to $b P$ as in the case where both detectors $D_{\mathrm{v}}$ and $\mathrm{D}_{\mathrm{t}}$ are APDs 7], a maximum of $f_{\mathrm{SNR}}(P)$ is achieved near a pump power $P_{\max }$ which gives the maximum conversion efficiency, and the maximum of $f_{\mathrm{SNR}}(P)$ is close to $f_{\mathrm{SNR}}\left(P_{\max }\right)=a /\left(b P_{\max }+d\right)$. On the other hand, when $d$ is sufficiently small as in the case of using the SSPDs, we may improve $f_{\mathrm{SNR}}(P)$ significantly by decreasing the pump power. Using $d / b \approx 3.3 \mathrm{~mW}$ in Fig. 2 and $\eta \tau^{2} \approx 3.6 \mathrm{~W}^{-1}[7]$, we see that $f_{\mathrm{SNR}}(P)$ reaches its maximum when the pump power is decreased from $P_{\max } \approx 700 \mathrm{~mW}$ to $50 \mathrm{~mW}$. In the following experiments, we chose the pump power to be $160 \mathrm{~mW}$, for which the conversion efficiency is not severely degraded (about half the maximum value) and the signal-to-noise ratio is expected to be above 10 .

Before the wavelength conversion of the entangled photon pairs, we measured the variance of jitter in the arrival time of pico-second photons to see the high timing resolution of the SSPDs. When we used the APDs for both detectors, the full width at half maximum (FWHM) of

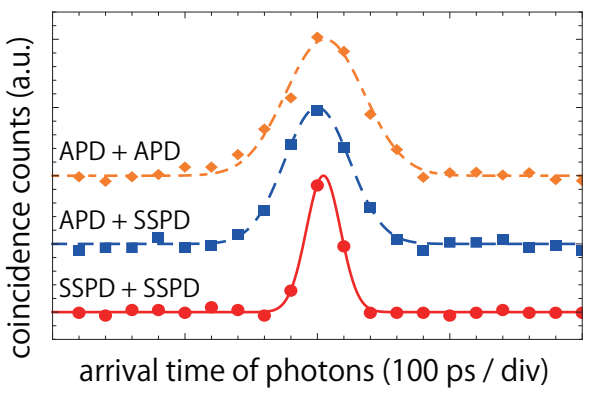

FIG. 3: The coincidence counts of the photon pairs recorded by the TDC with the timing resolution of $100 \mathrm{ps}$ when we used the pair of the silicon APD for $D_{v}$ and InGaAs/InP APD for $\mathrm{D}_{\mathrm{t}}$ (rhombus), the silicon APD for $\mathrm{D}_{\mathrm{v}}$ and the SSPD for $\mathrm{D}_{\mathrm{t}}$ (square), and the SSPDs for both the detectors (circle). Each result of the coincidence counts was fitted by the Gaussian function after the subtraction of the counts from the background noises. a

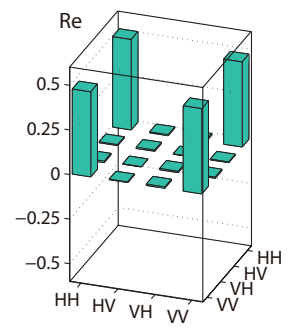

b

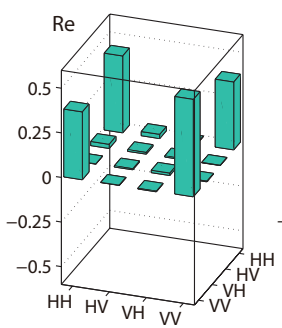

c

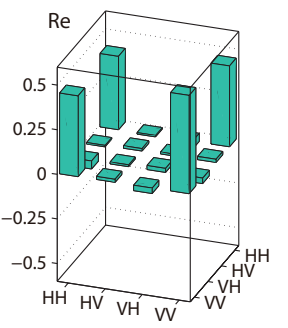

FIG. 4: The real parts of the reconstructed density matrices. (a) The initial entangled photon pair $\rho_{\mathrm{AB}}$ prepared by the BBO crystal. (b) The two-photon state $\rho_{\mathrm{AC}^{\prime}}^{\mathrm{AS}}$ after the wavelength conversion when we used the silicon APD for $D_{v}$ and the SSPD for $D_{t}$. (c) The two-photon state $\rho_{\mathrm{AC}^{\prime}}^{\mathrm{SS}}$ after the wavelength conversion when we used the SSPDs for both detector $\mathrm{D}_{\mathrm{v}}$ and $\mathrm{D}_{\mathrm{t}}$.

the time distribution of the coincidence counts was measured to be $\approx 350 \mathrm{ps}$. When we replaced the $\mathrm{InGaAs} / \mathrm{InP}$ APD with the SSPD for $\mathrm{D}_{\mathrm{t}}$, the FWHM was measured to be $\approx 290$ ps. When we used the SSPDs for both detector, the FWHM became $\approx 150$ ps. These results which are shown in Fig. 3 clearly show that the SSPDs gather the coincidence counts in the smaller time bins. The use of the SSPDs instead of the APDs is expected to improve the ratio of the signal photons to the optical noises by a factor of about 1.8 in our case of 200-ps time window.

In the experiment of the frequency down-conversion of the visible entangled photon pairs, we first performed quantum state tomography of the initial photon pairs in modes $\mathrm{A}$ and $\mathrm{B}$ at $780 \mathrm{~nm}$ by rotating a quarter-wave plate (QWP) and a HWP followed by a PBS in each mode 31. We used a silicon APD for $D_{v}$. The PMF before the frequency down-converter was connected to a silicon APD for detection of photons in mode B. We ob- 


\begin{tabular}{c|cccc} 
& $F$ & EOF & purity & $S$ \\
\hline$\rho_{\mathrm{AB}}$ & $0.97 \pm 0.01$ & $0.97 \pm 0.03$ & $0.97 \pm 0.02$ & $2.73 \pm 0.02$ \\
$\rho_{\mathrm{AC}}^{\mathrm{AS}}$ & $0.87 \pm 0.06$ & $0.68 \pm 0.15$ & $0.79 \pm 0.08$ & $2.35 \pm 0.10$ \\
$\rho_{\mathrm{AC}}^{\mathrm{SS}}$ & $0.93 \pm 0.04$ & $0.88 \pm 0.10$ & $0.93 \pm 0.07$ & $2.62 \pm 0.09$ \\
$\rho_{\mathrm{AC}}^{\prime}[7]$ & $0.75 \pm 0.06$ & $0.36 \pm 0.13$ & - & -
\end{tabular}

TABLE I: The observed fidelities, the EOFs the purities, and the $S$ parameters of the reconstructed operators before and after the wavelength conversion. $\rho_{\mathrm{AC}}^{\prime}$ is a reconstructed operator of photons in modes $\mathrm{A}$ and $\mathrm{C}^{\prime}$ by using the silicon $\mathrm{APD}$ and the InGaAs/InP APD for $\mathrm{D}_{\mathrm{v}}$ and $\mathrm{D}_{\mathrm{t}}$, respectively 7]. The attached errors are the standard deviations (1- $\sigma)$ with the assumption of the Poisson statistics of the counts.

served the two-photon state in modes $\mathrm{A}$ and $\mathrm{B}$ with a detection rate of $444 \mathrm{~Hz}$. Using the iterative maximum likelihood method [32], the density operator $\rho_{\mathrm{AB}}$ was reconstructed as shown in Fig. 团(a). From the reconstructed $\rho_{\mathrm{AB}}$, we calculated the fidelity defined by $\left\langle\phi^{+}\left|\rho_{\mathrm{AB}}\right| \phi^{+}\right\rangle$, the entanglement of formation (EOF) 33], and the purity defined by $\operatorname{tr}\left(\rho_{\mathrm{AB}}^{2}\right)$ as $0.97 \pm 0.01,0.97 \pm 0.03$ and $0.97 \pm 0.02$, respectively. These results show that the 780-nm photon pair before the wavelength conversion was highly entangled. Next, we performed quantum state tomography of the photon in mode $\mathrm{A}$ and the converted photon in mode $\mathrm{C}^{\prime}$ when we used the silicon APD for $\mathrm{D}_{\mathrm{v}}$ and the SSPD for $D_{t}$. An observed detection rate of the two photons was $0.324 \mathrm{~Hz}$ including background noises at a rate of $0.039 \mathrm{~Hz}$. The reconstructed density operator $\rho_{\mathrm{AC}}^{\mathrm{AS}}$ is shown in Fig. 团 (b). The fidelity, EOF and the purity are shown in Table \. We then switched the silicon APD to the SSPD for $D_{v}$, and performed quantum state tomography. In this case, we detected the two photons after the conversion at a rate of $0.280 \mathrm{~Hz}$ including noise photons at a rate of $0.015 \mathrm{~Hz}$. The reconstructed density operator $\rho_{\mathrm{AC}^{\prime}}^{\mathrm{SS}}$ is shown in Fig. 田(c). The observed fidelity, EOF and the purity are shown in Table \. We also performed the tests of the Clauser-Horne-Shimony-Holttype Bell's inequality 34 by observing the $S$ parameter. It is known that any local hidden variable theory leads to $S \leq 2$. The experimental results of the $S$ parameters are shown in Table प. The Bell's inequality was clearly violated with over 6- $\sigma$ deviation. From these results, we see that the high-fidelity frequency down-conversion was achieved, which could be more clearly observed with SSPDs.

In conclusion, we have demonstrated the faithful solidstate-based frequency down-conversion of visible photons at $780 \mathrm{~nm}$ to a telecommunication wavelength of 1522 $\mathrm{nm}$. Thanks to the lower dark count rates and the smaller timing jitters of the SSPDs, the observed fidelity of the two-photon state after the wavelength conversion was $0.93 \pm 0.04$, which was very close to the value of the fidelity obtained before the conversion. In this experiment, while the suppression of the effect of the Raman scatter- ing for the faithful wavelength conversion was achieved at the cost of the conversion efficiency, it will be attained without decreasing the conversion efficiency by a faster time-resolution measurement. Our demonstration shows the possibility of a noiseless and wide-band solid-statebased frequency down-conversion. We believe that such a quantum interface is vital for building quantum networks based on repeaters and for performing various quantum communication protocols.

This work was supported by the Funding Program for World-Leading Innovative R \& D on Science and Technology (FIRST), MEXT Grant-in-Aid for Scientific Research on Innovative Areas 20104003 and 21102008, the MEXT Global COE Program, and MEXT Grant-in-Aid for Young scientists(A) 23684035.

\section{REFERENCES}

[1] P. Kumar, Opt. Lett. 15, 1476 (1990).

[2] S. Tanzilli et al. Nature 437, 116 (2005).

[3] H. Takesue, Phys. Rev. Lett. 101, 173901 (2008).

[4] M. T. Rakher, L. Ma, O. T. Slattery, X. Tang, and K. A. Srinivasan, Nature Photonics 4, 786 (2010).

[5] H. J. McGuinness, M. G. Raymer, C. J. McKinstrie, and S. Radic, Phys. Rev. Lett. 105, 093604 (2010).

[6] Y. O. Dudin et al. Phys. Rev. Lett. 105, 260502 (2010).

[7] R. Ikuta et al., Nature Commun. 2, 537 (2011).

[8] S. Ramelow, A. Fedrizzi, A. Poppe, N. K. Langford, and A. Zeilinger, Phys. Rev. A 85, 013845 (2012).

[9] S. Zaske et al., e-print arXiv:1204.6253.

[10] S. Ares, I. Agha, A. Badolato, and K. Srinivasan, e-print arXiv:1205.2218

[11] H. -J. Briegel, W. Dür, J. I. Cirac, and P. Zoller, Phys. Rev. Lett. 81, 5932 (1998).

[12] L. M. Duan, M. D. Lukin, J. I. Cirac, and P. Zoller, Nature 414, 413 (2001).

[13] N. Gisin, and R. Thew, Nature Photonics 1, 165 (2007).

[14] J. I. Cirac, and P. Zoller, Nature 404, 579 (2000).

[15] D. N. Matsukevich, and A. Kuzmich, Science 306, 663 666 (2004).

[16] W. Rosenfeld et al., Phys. Rev. Lett. 101, 260403 (2008).

[17] C. W. Chou et al., Nature 438, 828.

[18] S. Olmschenk et al., Science 323, 486 (2009).

[19] E. Togan et al., Nature 466, 730 (2010).

[20] S. Ritter et al., Nature 484, 195 (2012).

[21] A. Politi, M. J. Cryan, J. G. Rarity, S. Yu, and J. L. OfBrien, Science 320, 646 (2008).

[22] K. F. Reim et. al., Nature Photonics 4, 218 (2010).

[23] E. Saglamyurek et. al., Nature 469, 512 (2011).

[24] H. Takesue, Phys. Rev. A 82, 013833 (2010).

[25] N. Curtz, R. Thew, C. Simon, N. Gisin, and H. Zbinden, Opt. Express 18, 22099 (2010).

[26] S. Zaske, A. Lenhard, and C. Becher, Opt. Express 19, 12825 (2011).

[27] S. Miki, M. Takeda, M. Fujiwara, M. Sasaki, and Z.Wang, Opt. Express 17, 23558 (2009).

[28] S. Miki, T. Yamashita, M. Fujiwara, M. Sasaki, and Z. Wang, Opt. Lett. 35, 2133 (2010). 
[29] T. Yamamoto, K. Hayashi, Ş. K. Özdemir, M. Koashi, and N. Imoto, Nature Photonics 2, 488 (2008).

[30] T. Nishikawa et al., Opt. Express 17, 17792 (2009).

[31] D. F. V. James, P. G. Kwiat, W. J. Munro, and A. G. White, Phys. Rev. A 64, 052312 (2001).

[32] J. Řeháček, and Z. Hradil, Phys. Rev. A 75, 042108
(2007)

[33] W. K. Wootters, Phys. Rev. Lett. 80, 2245 (1998).

[34] J. F. Clauser, M. A. Horne, A. Shimony, and R. A. Holt, Phys. Rev. Lett. 23, 880 (1969). 